\title{
Navegando pela Cooperativa: percepção e expectativas dos usuários*
}

Cleci Maraschin

Elis,ngela Zaniol

\section{Surfing the Cooperative: perceptions and expectations of users}


Resumo: Este projeto decorre de uma sÈrie de questıes derivadas de pesquisas desenvolvidas nos 'Itimos dois anos pelas autoras. $O$ atual estudo apoiar. a construÁ,,O e avaliaÁ,, de um ambiente virtual de interaÁ,,o denominado de Cooperativa do Conhecimento, no projeto coordenado pelo LaboratÛrio de Estudos Cognitivos da UFRGS, em parceria com a SMED/POA. A Cooperativa, que est. em fase inicial de implementaÁ,,o, ser-um ambiente virtual catalizador dos projetos de aprendizagem de professores e alunos da rede municipal de ensino. Realizamos ent,,o um estudo piloto, para auxiliar no desenvolvimento de espaÁos virtuais de interaÁ,o dentro da Cooperativa. Foi possìvel identificar modos interativos relacionados a sensaÁ,o de imersividade dos estudantes quando exploraram o ambiente da cooperativa. A imers „o È conceituada como efetivaÁ,,o de um acoplamento estrutural de acordo com o referencial da Biologia do Conhecer. Os sujeitos escolhidos s,,o alunos da rede municipal de ensino. Analisamos como as interaÁıes dos sujeitos nestes ambientes podem se constituir em experiĺncias imersivas, buscando avaliar que elementos devem ser implementados, preservados, desativados no ambiente. Os dados obtidos atravĖs foram organizados e analisados atravĖs do software CHIC.

Palavras-chave: ambientes imersivos; realidade virtual e educaÁ,,o, interaÁ, „O.

\begin{abstract}
This project resulted from a series of inquiries derived from researches developed in the last two years by the authors. The current study will support the construction and evaluation of a virtual environment of interaction called the Cooperative of Cognition, in the project coordinated by the Laboratory of Cognitive Studies (LaboratÛrio de Estudos Cognitivos) of the Federal University of Rio Grande do Sul (Universidade Federal do Rio Grande do Sul), in partnership with the Municipal Secretary of Education (Secretaria Municipal de EducaÁ,,o) of Porto Alegre, Brazil. The Cooperative, which is in the initial phase of implementation, will be a catalizing virtual environment for learning projects of teachers and students of the municipal teaching network. We accomplished a pilot study, to aid in the development of virtual spaces of interaction in the Cooperative. It was possible to identify interactive manners related to the studentís sensation of immergence when they explored the virtual environment of the cooperative. Immergence is considered as effective structural coupling in accordance with the referred Biology of Cognition. The subjects chosen were students from the municipal teaching network. We analyzed how the subjectís interactions in these environments can be constituted in immersible experiences, searching to evaluate which elements should be implemented, preserved, disabled in the environment. CHIC software was used to organize and analyze the data.
\end{abstract}

Key-words: immersible environments, virtual reality and education, interaction.

Maraschin, Cleci; Zaniol, Elis,ngela. Navegando pela Cooperativa: percepÁ,,o e expectativas dos usu.rios. Informática na Educação: teoria \& prática, Porto Alegre, v.7, n. 1, p. 73-85, jan./jun. 2004. 


\section{Introdução}

Este estudo analisa os modos de interaÁ,,o com um ambiente de realidade virtual de estudantes do ensino fundamental de escolas P'blicas da Rede Municipal de Porto Alegre, vinculadas ao Projeto Escola, Conectividade e Sociedade da Informação' ${ }^{1}$, com vistas a uma definiÁ,,o das condiÁıes de imersividade da interaÁ,,O. O projeto Escola, Conectividade e Sociedade da InformaÁ,o propıe o desenvolvimento projetos de aprendizagem em ambientes informatizados na totalidade das escolas da rede municipal de ensino. A Cooperativa do Conhecimento pretende ser um ambiente virtual catalisador dos projetos de alunos e professores propiciando a troca e a interaÁ,,o, com o objetivo de problematizar os conte dos trabalhados atravÈs do debate de idÈias, de novas propostas e desafios.

O projeto atual problematiza a relaÁ,,O entre a imersividade e ambientes de realidade virtual dando continuidade a uma sÈrie de questies levantadas em estudos desenvolvidos no Projeto ARCA,${ }^{2}$ no qual as autoras participaram da equipe de pesquisa. O Projeto ARCA propunha o desenvolvimento de um ambiente de aprendizagem via Web,que, utili- zando diferentes recursos e ferramentas computacionais de realidade virtual, apoiasse a cooperaÁ,,o entre os participantes propiciando a constituiÁ,,o de um ambiente virtual de aprendizagem. Esperava-sequeesseambiente fosse imersivo, ou seja, que o sujeito pudesseinteragircomomesmode modoquetivesse uma crenÁa de genul̀na de participaÁ,,o e interaÁ,,o.

Autores que abordam o tema divergem a cerca das relaÁıes entre Realidade Virtuale imers,,o. Para alguns, como Psotka, citado por Casas (1999), a realidade virtual imersiva dependedos equipamentos parainteraÁ,,ocomo computador. UmainteraÁ,,oresultaimersivade acordo com a capacidade que o computador tem de detectar as entradas do usu-rio e modificar instantaneamente o mundo virtual e as aÁıessobre ele. Esses autores acreditamque para que haja a imers,,o È necess.rio utilizar equipamentosperifĖricossensoriaiscomoÛcuIos, luvas e capacetes. Ou seja, a imers „, o seriadependente das condiÁıes doambiente.

Em uma outra linha de raciocl̀nio, a imers, ,o pode ser definida como ìa simulaÁ,,o de meio-ambientes e dos mecanismos sensoriais do homem pelo computador, de maneira tal que busca proporcionar ao usu -rio a sensaÁ,,o de imers,,o e a capacidade de

\footnotetext{
${ }^{1}$ Coordenado pelo LaboratÛrio de Estudos Cognitivos (LEC), do Departamento de Psicologia Social e Institucional da UFRGS, em parceria com a Secretaria Municipal de EducaÁ,o de Porto Alegre, se realiza desde 2002. Com este estudo, apoiamos a construÁ,,o e avaliaÁ,,o de um ambiente virtual de interaÁ,,o denominado de Cooperativa do Conhecimento <http://www.curie.psico.ufrgs.br/coop>.

${ }^{2} \mathrm{O}$ projeto ARCA trata-se de um projeto multidiscilplar formado pelas universidades: Universidade Luterana do Brasil, Universidade CatÛlica de Pelotas e Universidade Federal do Rio Grande do Sul. Na sua primeira vers,„o, o ARCA partiu da criaÁ,,o de diferentes objetos (prÈdios, plantas, etc.) para chegar $\ddagger$ simulaÁ,o de dois laboratÛrios de alimentos, no ambiente denominado UCPel. Nestes, os usu.rios, alunos do curso de engenharia de alimentos da UFRGS, foram desafiados a fim de aprender e compreender os fenÙmenos que regem as relaÁıes de deterioraÁ,o e conservaÁ, ,o destes materiais. Recentemente, os 'Itimos testes feitos no mundo, levaram $¥$ criaÁ, o de novos laboratÛrios, totalizando quatro: dois para continuar as experí́ncias na rea de engenharia de alimentos, um para experiĺncias na rea de fl̀sica e outro na rea de redes. Maiores informaÁıes sobre o projeto ARCA podem ser encontradas em <http://www.pgie.ufrgs.br/projetos/arca>.
} 
interaÁ,ocommeio-ambientes artificiaisînios (em Casas, 1994). Arlindo Machado (1996), discute a imers, , o cinematogr.fica em que, em seu estado pleno, o espectador poderia ientrar no filme, atravessar a fronteira entre o real e o virtual, passar para o lado de I., escapar para dentro do universo de pura ficÁ „, do cinema...i sem necessariamente o uso de equipamentos perifĖricos acoplados. Para estes autores a realidade virtual imersiva $n, 0$ depende de equipamentos especiais massim da interaÁ,, 0 do sujeito com dispositivos de simulaÁ,,o. O fenÙmeno da imers,,o implica um acontecimento entre o sujeito da aÁ,,o e as condiÁıes do ambiente.

No intuito de estudararelaÁ,ointerativa de sujeitos e as condiÁıes de ambientes de realidade virtualj: produzimos alugns estudos anteriores (Maraschin,CeMesquita, J.L.2000; Maraschin, C.eZaniol, E,2001; Maraschin, C.; Schuch, E.;Zaniol, E; Tomaz, A. R, 2002). Os resultados possibilitaram evidenciar a import,ncia dos estudos da percepÁ,,o para a compreens „, o do fenÙmeno daimers,,O. Aimers,,O seria advinda da organizaÁ „o de um espaÁo perceptivo inusitado decorrente de novas congruÍncias sensûrio-motoras e simbÛlicas na relaÁ,,o entre o sujeito e o ambiente. Para Maturana (1994) a percepÁ,,o È advinda do quechamade configuraÁ,,odecondutadosobjetos. Os objetos que povoam nosso universo fìsicoesimbÛlico, estruturam-se, n,,oprË-existem $\ddagger$ aÁ,,o do observador. O que reconhecemos como objetivo $\mathrm{S}$, , O as configuraÁıes de conduta que realizamos para distinguir determinado objeto de umfundo. Aestabilidadedessas relaÁles de distinÁ,,o e sua recursividade no coletivo explicam porque vivemos em um mundo objetivo e com const,ncias. A idĖia de algo externo agindo sobre o sujeito sû È possìvel porqueh-um terceirolugar, ode umobservador,queví, atravĖs docomportamentodede- terminado sujeito, que este respondeu a algo queestavaemseucampoperceptivo.Mas, para o organismo que interage o que temos s,, acoplamentosestruturais

Em lugar de representar um mundo independente, ossistemas cognitivosconstroem um mundo como um domìnio de distinÁıes, insepar-vel das estruturas de nossa corporeidadesensÛrio-motora. Omundodosobjetos È resultante ent,,o de um processo de construÁ,o, de criaÁ,,o de uma forma, a partir de uma rede de configuraÁ, ,o heterogíneaque incluia estrutura do sistemanervoso, dos Ûrg,,ossensoriais, a histÛria de acoplamentos, a linguagem, as tecnologias, as instituiÁıes sociais. Desta forma, possulmos tantos espaÁos perceptivosquantoclasses de correlaÁıessensÛrio-motoras possamos realizar a partir da interaÁ,,O com as diversas configuraÁıes da rede mencionada. Esta 'Itima constataÁ,,ofaz com que seja possìvel pensar que a interaÁ,,O com ambientes virtuais possa vir a constituir outras regularidades e congruĺncias perceptivas de tal modo que possibilite a constituiÁ,,O deoutrosobjetos perceptivos decorrentes dessas mesmas configuraÁıes de conduta recorrentesecongruentes.

Oambientevirtual poder-serexplorado, vivenciado, comoumespaÁo perceptivo inusitado ao serem produzidas congruĺncias sensÛrio-motorase simbÛlicas entresujeitos/usuriosquealiinteragem. OfenÙmenodaimers, , indica um efeito de acoplamento, ou seja, um acoplamento efetivo produz a sensaÁ,,o de imers,,o.

Neste estudo, nos propomos identificar emque medida, as interaÁıses em umambiente de realidade virtual podem se constituir como uma experíncia em que os sujeitos se sintam imersos e quais $\mathrm{s}, \mathrm{O}$ as caracterìsticas dessa interaÁ,,oque podem se constituir emoperado- 
resdeacoplamentonesseambiente. Essaques$\mathrm{t}$, ,ocontÈmum significativo interesse nocampo daintroduÁ,,odas novastecnologiasnaeducaÁ,o,j-quesediscuteoquantoumambienteinfor$\mathrm{m}$-ticodeaprendizagemeducacional podefuncionarcomoumpossibilitadordeaprendizagem.
Oestudodascaraterl̀sticasimersivas da interaÁ,,o de estudantes no ambiente da cooperativautilizou comoindicadoresanalìticosas categorias definidas em estudo anterior (Maraschinetal.,2002):

Modos de interaÁ, o

1. Domìnio da interface

2. ProduÁ, o de contextos de aÁ, o

3. Familiaridade com o real

4. Estranhamento e ambig, idade

5. Interatividade

6. Objetivo de longo prazo

7. Ludicidade

8. Plasticidade do ambiente

9. Interesse de exploraÁ, o
ExploraÁ, o de aÁ es que possibilitem a navegaÁ, o, exploraÁ, o e a aÁ, o no ambiente.

ConstruÁ, o de uma relaÁ, o entre a experí ncia atual no ambiente com conhecimentos anteriores; relaÁ es com outras situaÁ es, com alguma experiĺ ncia j. passada pelo sujeito na vida real ou no uso da Internet.

ProduÁ, o de analogias com ambientes materiais tanto em relaÁ, o ‡ apresentaÁ, o gr. fica ou IÛ́gica do ambiente.

ManifestaÁ, o de estranheza, descontentamento ou perplexidade em relaÁ, o a situaÁ es ou propostas do ambiente.

Se o sujeito procura descobrir lûgicas internas ao ambiente ou se comunicar com outros sujeitos.

Se o sujeito vislumbra aÁ es a serem perseguidas na exploraÁ, o do ambiente ou se tem ou manifesta intenÁ, o de entrar/atuar no ambiente novamente.

Se sujeito se sentiu atraldo pelo ambiente, se a sua exploraÁ, o foi prazerosa.

Se o sujeito percebe que o ambiente pode sofrer influí ncia da sua prûpria aÁ, o.

Se o sujeito procurou descobrir detalhes de funcionamento, organizaÁ, o, composiÁ, o, arquitetura do ambiente sem que fossem feitas observaÁ es de incentivo para esta atitude.

Quadro 1: CaracterizaÁ,o dos modos de interaÁ„,o

Os resultados possibilitaramidentificar pontosdeacoplamentoondenovas regularidades poderiamoun,,oserestabelecidas. Funcionam como descritores que possibilitam pensar na implementaÁ,,o e transformaÁ,,o dos desenhos dos ambientes. Pensamos que ao incrementar a ocorríncia desses pontos de interaÁ,o seria possìvel incrementar a imersividade. Os resultadostambÈmpossibilitaram constituir um modelo de avaliaÁ,,o da 
imersividade dos ambientes. Nestaan.lise vamos tomaros modos de interaÁ,,onoambiente como observ veis da possibilidade de imersividade. Seaapostado projeto nas escolas Ė a aprendizagem dos alunos, a imersividade nos ambientes seria uma condiÁ,,o necess-riapara sua ocorríncia. Um outro aspectodecorrentedas experínciasanterioreseque È tambÈm significativo para o atual projeto È como ambientes imersivos potencializam a interaÁ „, e a cooperaÁ,,o entre estudantes, independentemente da proximidade geogrfica, constituindo redes diferenciadas de aprendizagem.

\section{Método}

Trata-se de uma pesquisa exploratÛria que busca analisar as possibilidades de imersividade na interaÁ,,o com ambiente de realidade virtual denominado Cooperativa do Conhecimento.

Durante o processo dessa an-lise, a Cooperativa encontrava-se tambÈm em uma fase incipiente de desenvolvimento. Os resultados do estudo contribul̀ram para propor alteraÁıes na mesma. A Cooperativa est. sendodesenvolvidaatravĖsdatecnologia Flash comoutros recursos da Web.

No momento a cooperativa apresenta, como projeto, v rios espaÁos de interaÁ,o. Sua tela inicial È denominada de Cidade, nela est,"o representados alguns pontos turìsticos e marcos da cidade de Porto Alegre. Estap.gina servir - de İndice de links paraprojetos deaprendizagem desenvolvidos poralunos e professores das escolas. Dependendo da tem tica do projeto de aprendizagem ele poder-serìlinkadoîemumterritÛrio especl̀fico dacidade. AopÈdap.ginaexisteum menucontendo as palavras em login, clube, condomìnio, escola, jogos, postais, buscadoremapado site.
Est,,o somente implementados: o Clube, os Jogos e o Buscador. O Clube, previsto para cadastro e encontro dos membros da comunidade, onde existe a proposiÁ,,o de constituiÁ,,o de grupos tem ticos de interesse entre os participantes, troca decartıes, jogos.

Abaixo algumas imagens da Cooperativa:

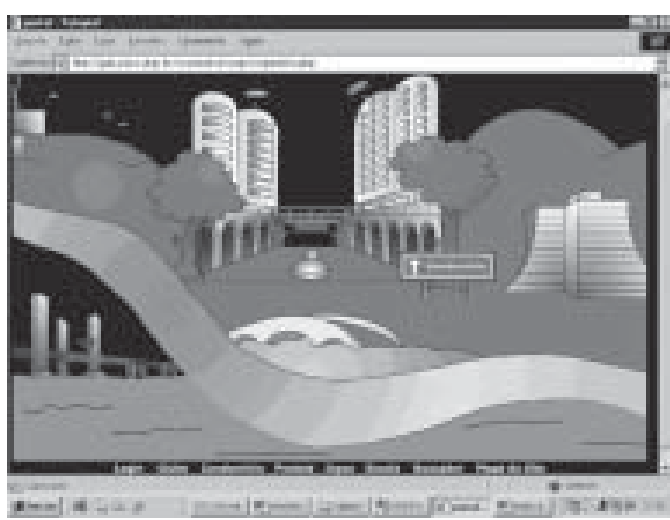

Figura 1:Tela inicial

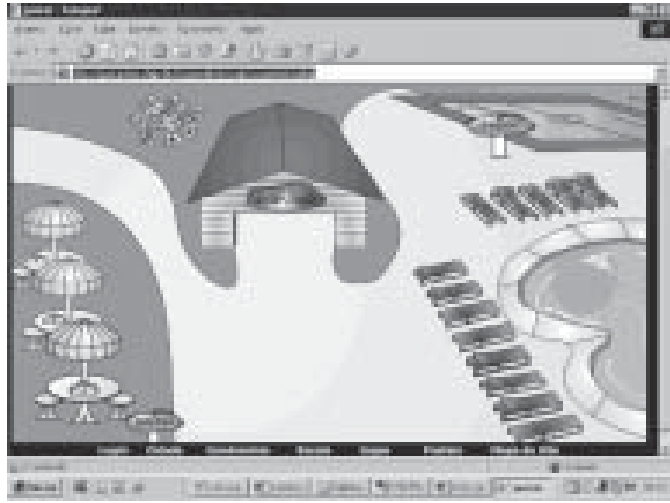

Figura 2: Clube

Na Figura 1 podemos observar a imagem dap.gina de navegaÁ,o inicial da Cooperativa do Conhecimento. No Clube, um espaÁo previsto paraserde interaÁ,,o e trocas entre alunos das diferentes escolas municipais. No momento È apenas possìvel navegar pelo ambiente. 

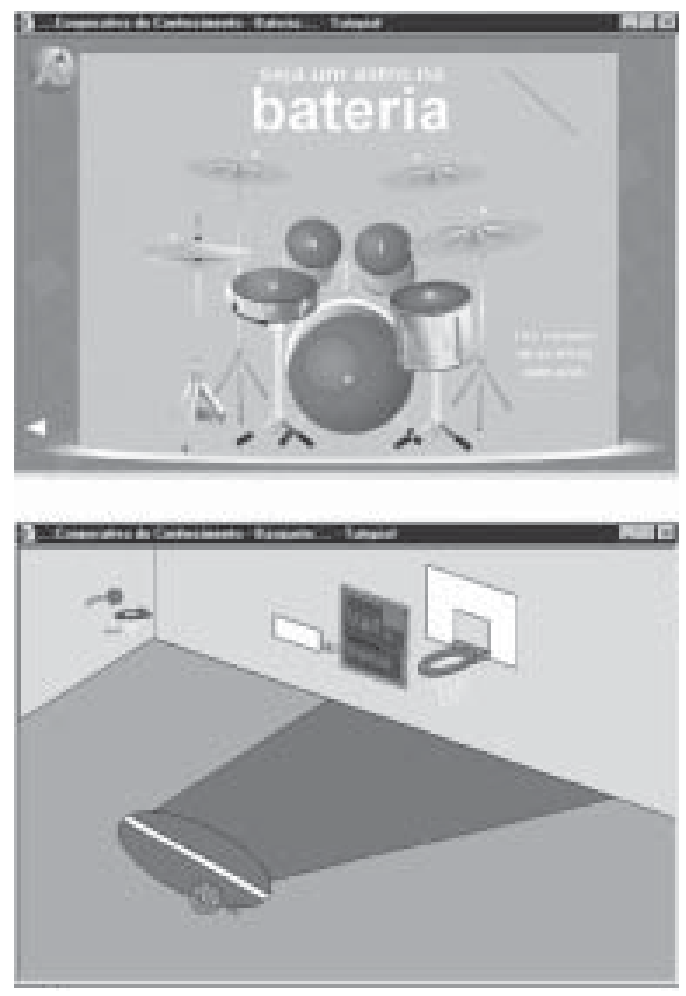

Figura 3: Jogos disponl̀veis na Cooperativa do Conhecimento

Os Jogos, onde È possìvel encontrar jogoscomoping-pong, basquete, xadrezeuma bateria onde digitando letras, ou usando o mouse pode-se ouvir o som digital do instrumento.

OBuscador, tambÈmconhecidocomo Weblec, um portalonde È possìvelfazerbusca em sites a respeito de conte "dos desenvolvidosnas disciplinas dasescolas. Apartirdeuma busca inicial, os sites s, o categorizados por assunto, pal̀s de origeme lìngua.

\subsection{Sujeitos e Coleta de dados}

Convidamos, ent,o, vinteestudantes do ensino fundamental detrís escolas municipais de Porto Alegre para explorar o ambiente da CooperativadeConhecimento:EscolaMunici-

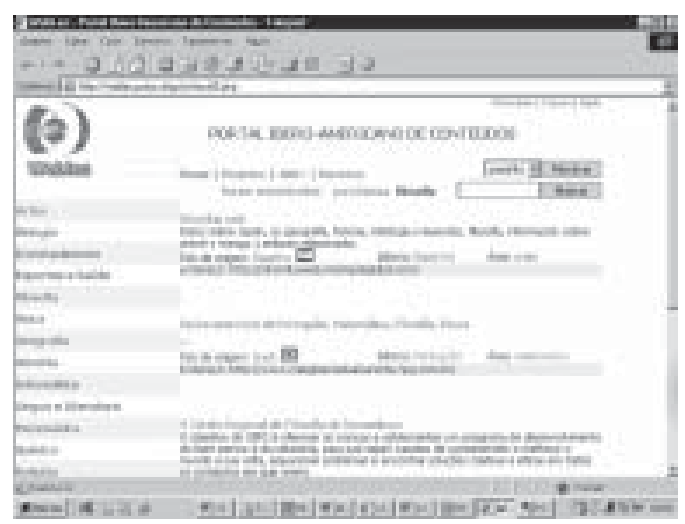

Figura 4: Imagem da p.gina do Buscador

pal Nossa Senhora de F-tima, Escola MunicipalM-rio QuintanaeEscolaMunicipalCampos deCristal. Afaixadeidadedosestudantes, est. compreendida entre oito e treze anos, sendo desses, onze sujeitos masculinos enove femininos. Cadaestudante permaneceu explorando o ambiente, em mÈdia, por 18 minutos. $O$ tempo de permaníncia em cada espaÁo da Cooperativafoicronometrado, as falas durante aexploraÁ,,oforamregistradas. ApÛsaexploraÁ,,o do ambiente, procedeu-se a uma conversaÁ,,oindividual baseadanomÈtodo clìnico, no intuito de explorar o que pensaram do espaÁo da cooperativa, como se sentiram ao explor-la, qual caracterlstica lhes parecia mais interessante, qual lhes parecia mais estranha, se nessa exploraÁ,,o encontraram algo parecido com outra experiĺncia vivida, que elementos acrescentariam se pudessem construir acooperativa.

\subsection{Análise de Relações entre os Modos de Ação}

OsdadoscolhidospossibilitaramaidentificaÁ,,o das nove categorias, modos de interaÁ,,o, que foram observadas tanto pelos sujeitos durante os di-logos posteriores a interaÁ,,o quanto pela investigadora durantea 
Tabela 1

Sexo, idade e permanÍncia dos sujeitos na Cooperativa

\begin{tabular}{lcccccccccccccccccccccc}
\hline Sujeitos & S1 & S2 & S3 & S4 & S5 & S6 & S7 & S8 & S9 & S10 & S11 & S12 & S13 & S14 & S15 & S16 & S17 & S18 & S19 & S20 \\
\hline Sexo & M & M & M & M & F & F & F & M & M & M & F & M & M & F & F & M & F & F & M & F \\
\hline $\begin{array}{l}\text { Idade } \\
\text { Permaní ncia }\end{array}$ & 13 & 14 & 15 & 16 & 13 & 13 & 15 & 12 & 12 & 14 & 16 & 14 & 12 & 9 & 10 & 14 & 12 & 8 & 16 & 14 \\
$\begin{array}{l}\text { Permambiente } \\
\text { (min) }\end{array}$ & 19 & 20 & 20 & 20 & 7 & 15 & 20 & 20 & 20 & 9 & 20 & 16 & 20 & 15 & 20 & 17 & 9 & 13 & 12 & 17 \\
\hline
\end{tabular}

observaÁ,o da exploraÁ,,o no ambiente. Essas categorias podem operar como possibilitadoras ou restritoras de um acoplamento efetivo que aqui estamos definindo como imers, ,o.

Para essa an-lise procedemos da seguinte forma: a presenÁa ou a ausíncia dessas condutas foi identificada nas interaÁres e falas dos sujeitos. Foram construldas matrizes de dupla entrada em que os valores eram ou positivos (1), referentes ł presenÁa da conduta; ou nulos $(0)$, referentes $\ddagger$ ausíncia da conduta. De posse de uma matriz procedemos a uma an -lise das relaÁres de implicaÁ,,o e de similaridade entre as categorias atravÈs do software CHIC (Classification Hierarchique Implicative Et Cohésitive) Vers „, Windows 1.1 ñ01 de Setembrode 1999, produzido por Antoine Bodin, Raphael Couturier e Regis Gras, do Instituto de Matem ticada Universidade de Rennes 1

As relaÁıes de implicaÁ,,o e de similaridade das categorias possibilitam identificarque condutas, modos de interaÁ,,o ocorreram com mais proximidade equaisforam mais relevantes eque funcionaram como atratores deoutras.

Vejamos os gr.ficos gerados pelo softwareCHIC.

OGr.fico 1 permite constatarque parao grupodeestudantesque interagiramcomesse ambienteexisteumaredeimplicativaentreseus modos de interaÁ,,o: a possibilidade de construÁ,,o de objetivos a longo prazo com o ambiente Ė dependente da ludicidade. Os alunos manifestaram desejo de retornar ao ambiente ou de continuar explorando em torno de um objetivo especilfico, quando sentem curiosidade ao navegar, sentindo o ambiente como I'dico.

O gr.fico de implicaÁ,,o indica que o estranhamento que elementos do ambiente causam, somada ao doml̀nio da interface, $\mathrm{S}, \mathrm{O}$ duas caracterlisticas imprescindìveis paraque o ambiente seja percebido como I'dico. O estranhamento proporciona, alÈm da ludicidade, a produÁ,,o de contextos de aÁ, „O. Outros modos n,,o entraram na composí, „, da rede implicativa de um modo significativo.

O gr.fico de similaridade aparece dividido em duas grandes redes: a primeira contÈm modos de interaÁ, ,o com maior proximidade de ocorríncia simultaneamente. Esse resultado È muito semelhante ao do gr.fico implicativo analisado anteriormente. Existe umaaproximaÁ,,oentreoestranhamento, aformulaÁ,,O de objetivo de longo prazo e a ludicidade. O estranhamento aparece como uma caracterìstica capaz de manter o sujeito explorando o ambiente durante mais tempo, perseguindo objetivos de longo prazo, 0 que torna a exploraÁ,,o prazerosa. Nessa mesma rede de proximidades est, ,o a possibi- 
Gr.fico 1

ImplicaÁ,o entre os modos de aÁ,o na cooperativa
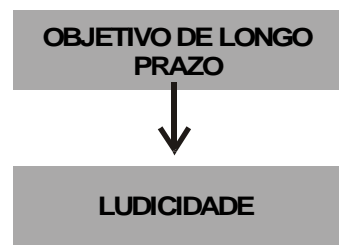

PRODUÇÃO DE CONTEXTOS DE AÇÃO
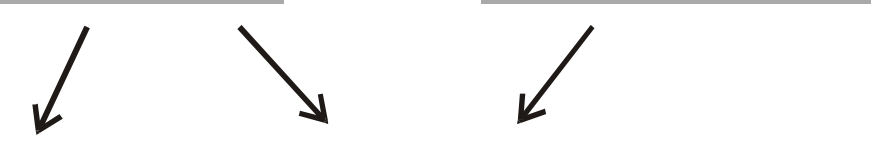

DOMÍNIO DA INTERFACE

ESTRANHAMENTO

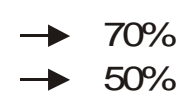

FAMUARIDADE

INTERESSE NA

EXPLORAÇÃO

\section{INTERATIVIDADE}

PLASTICIDADE

Gr.fico 2

Similaridade entre os modos de aÁ,,o na cooperativa

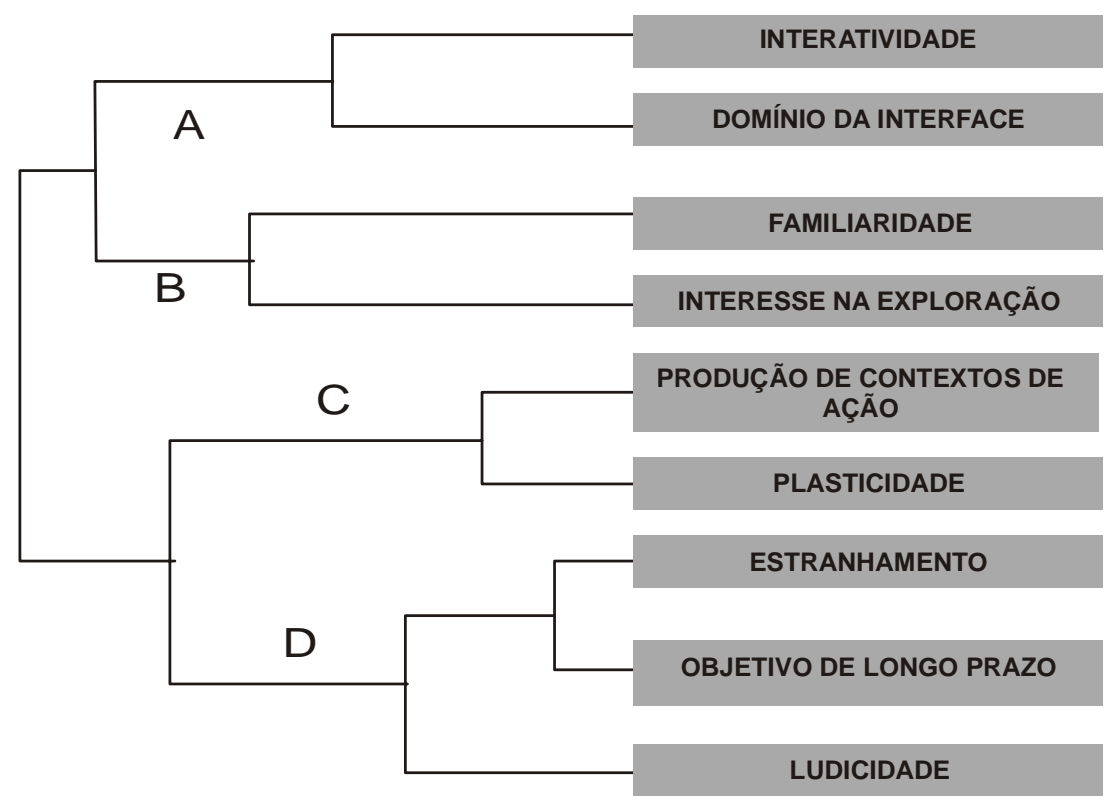


lidade de produÁ,,o de contextos de aÁ,,o e a plasticidade. Umambiente pl-stico, onde osujeito tema possibilidade de reconheceros efeitos da sua aÁ,,o no ambiente, facilita a produÁ,,o de contextos de aÁ,,o onde o sujeito pode construir relaÁıes entre a experiĺncia atual no ambientee os conhecimentos anteriores.

AsegundaredecontÈmcategorias relacionadas $\ddagger a A ́$, o no ambiente, em duas ramificaÁıes. NaramificaÁ,,o Aest,„o a interatividade eodoml̀niodainterfacea similaridade. Ainteratividadedava-seao mesmotempoque o sujeito explorava o ambiente, aprendendo a dominar a interface, devido a prÛpria facilidade da navegaÁ,,o. Na segunda ramificaÁ,,o, h· uma aproximaÁ, o dos conceitos de familiaridadee interesse pela exploraÁ,,o do ambiente.

\subsection{O que os estudantes esperam da Cooperativa}

A conversa individual com cada aluno, apÛs a exploraÁ,o do ambiente e suas manifestaÁıes notranscurso daprÛpria exploraÁ,,o, trouxe indicadores das suas expectativas em relaÁ,,o $\ddagger$ cooperativa. Umaparcela dosalunos (30\%) sugeriu a necessidade de colocar mais escolas e praÁas no ambiente da cooperativa. As escolas seriam como a do prÛprio aluno, com pavilhıes e salas para estudar, com refeitÛrioelanche. EpraÁasñespaÁosparasedeslocar como o calÁad,,o, espaÁos para praticar esportes, com brinquedos como balanÁo, escorregador, gangorra, gira-giraevai-e-vem.

Em vintee cincoporcento das opiniıes, os alunos disseram que gostariam de encontrar campos de futebol ñ sÛ o campo ñ, escolinhas defutebol, jogos interativosecasas para os pobres, interativas e equipadas com eletrodomÈsticosepessoascaminhando, pessoascomqueminteragir.
Dez por cento dos sujeitos sugeriram que o ambiente deveria ter: links para outras cidades; mais ruas; -rvores; outros esportes; crecheehospital;jogos ñde corrida, de montar, sinuca, sena; links para outros sites.

\section{Discussão}

As observaÁıes feitas durante a exploraÁ,,o dos alunos na Cooperativa corroboram os resultados das an-lises dos gr-ficos. Em funÁ,o do ambiente possuir uma sÈrie de elementosfamiliaresaossujeitos, principalmente pelainterface, fazcom que se torne def-cilexploraÁ,,o e compreens,„O. Aspectos como domìnio dainterface, interatividade, interesse na exploraÁ,,oforam rapidamente incorporados. Estacaracterl̀sticaproporcionavaqueossujeitos entendessemcomooambientefuncionava e em seguida exploravam-no como um todo, esgotando as possibilidades de interaÁ,,ocom o ambiente em menos de vinte minutos.

As falas possibilitaram observar que o que lhes causou maior estranhamento foram, ent,,o, as ìlacunasî que ocorreram durante a exploraÁ,,o do ambiente. Chamamos aqui de lacunas tanto os momentos em que os sujeitos tinham de se adaptar $¥ s$ dificuldades ñ por exemplo, velocidadedaconex,oñdaCooperativa, quanto a ausíncia de desafios do ambiente. Essas lacunas parecem ter impulsionado ainda mais para que os sujeitos falassem do queesperavamencontrarnoambiente, e, apartir dal̀, imaginavam situaÁıes em que se colocariam, produzindo contextos deaÁ,o, ou seja, buscandorelaÁıes entreoqueestavamexperimentando na exploraÁ,o com suas experiĺncias anteriores para falar do que esperavam encontrarno ambiente. A plasticidadedoambiente se constitula muito mais no plano da imaginaÁ,,o de possìveis aÁıes no ambiente. EsseÈ um fator que julgamos que possa ser impor- 
tante para um movimento de imers,,o: a produÁ,,o de outras possibilidades de aÁ,,o no ambiente. No caso, foi involunt-ria, ou seja, ocorreu devido ao est.gio ainda inicial de desenvolvimentodoambiente. Masestacircunst,nciapossibilitou enfatizar que as condiÁıes de fechamento e de definiÁ,,o total de um ambiente talvez levem a uma maior esgotabilidade da prûpriainteraÁ,,oe conseq,entemente daimers,,o.

O fato do ambiente possuir elementos familiares ao cotidiano dos sujeitos e estar em uma fase inicial de implementaÁ,o produzuma limitaÁ,,o no tempo de exploraÁ,o e na manutenÁ,,o dointeresse naprÛpria exploraÁ,,O. Em funÁ,odisso, podemos compreenderporquea familiaridade, o interesse na exploraÁ,,o, a interatividde e a plasticidade n,o apareceram significativamente implicadas com as demais condutas.

No gr.fico da similaridade È possìvel observar a constituiÁ,,o de duas redes: uma, referente ¥saÁıes de construÁ,,o e ludicidade noambiente, e, outra, relacionadaaodomìnioe ‡exploraÁ,,o. AcategorizaÁ,,o dasaÁıes, desta forma, pode produzir uma fronteira entre aÁ,,o produtivaeinterativa, onde explorare produzir podem ser pensados por uma relativa oposiÁ,,O. J. observamos que o est.gio do desenvolvimento do ambiente pode ter contribuldo para uma sensaÁ,o de pouco a explorar, mas tambÈm existe na escola uma sÈrie de restriÁıesłsatitudesexploratÛrias, deensaio-e-erro, porpartedosalunos, pois s, oconstantemente confrontados com modelos de resoluÁ,,O de problemas, comalgoritmosj. definidos. Caberia seguir investigando, se, nos est-gios mais avanÁados de desenvolvimento do ambiente, essamesmafronteirase reproduz.

As expectativas dos alunos em relaÁ,,O ao ambiente revelam que, apesar da familiaridade com alguns elementos, seria desej-vel maisproximidade. AreivindicaÁ,oprincipaldos estudantes È que a cooperativa seja um espaÁode maiorinteratividade, conectada comavivíncia dos mesmos, a partir de ativaÁ,o de elementos como a escolaedaimplementaÁ,,o de praÁas com brinquedos, jogos, onde seja possìvelinteragircomoutraspessoas. Essedesejo defamiliaridadecontrastacomoquevìnhamos discutindo:oinacabamentodoambiente, ouseja, apossibilidadedeexistínciadebrechasconvoca $\ddagger$ imaginaÁ,,oe $\neq$ proposiÁ,,o dealternativas.

\section{Conclusões}

Se a imers,,o È a efetivaÁ,,o de acoplamentos estruturais, podemos pensar que esseacoplamento ̇̀tantodependentedosestudantes quanto do ambiente de realidade virtual proposto. O fato de a interface apresentar facilidade de exploraÁ,o e de entendimento constitui-se em um indicativo favor-vel $\ddagger$ efetivaÁ,,odeumacoplamento. Outroindicador interessante È que a prÛpria exploraÁ,,o pode configurar-secomo arealizaÁ,,o de um percurso dentro de um roteiro. Confirma-seaposiÁ,,o de Assis (1999) para quem o roteiro de um ambiente de realidade virtual È a sua prûpria arquitetura...., atravĖs dela, que o sujeito se sente motivado a participar, entrando num enredo que lhe pareÁa suficientemente interessante (congruÍncia conceitual) para que n,o abandoneolocal nosprimeirosmomentos. Um roteiro aberto ñ pl-stico o suficiente para ser afeito ¥s interaÁıes de diferentes sujeitos ñ contemplaatividadesexploratÛriasquedesafiam a capacidade de fazer correlaÁıes significativas, permitindo que os usu-rios interajam para buscar soluÁıes compartilhadas. Podese dizerque a arquitetura da cooperativa comporta-secomo um roteiro. Natelainicial, podese ìpercorrerî lugares de Porto Alegre, entrar emclubee, dentro dele, realizaralgum jogo. 
A plasticidade do ambiente $\grave{E}$ algo que necessitar de maiores investimentos. Osestudantesn,o conseguem perceber a influÍncia das suas prÛprias aÁıes no ambiente. Foram convocados a outros possìveis dos $\mathrm{j}$ - atualizados, ao produzirem contextos deaÁ,,o com aquilo que imaginaram poder encontrar no ambiente.

A interatividade, em estudo anterior (Maraschinetal.,2002), apareceucomoumponto muito relevante para a imers,,O, seja atravĖs do roteiro dos ambientes que pode funcionar comoummotivadorparaaexploraÁ,,o, sejaatravĖsdautilizaÁ,odospersonagensparainteragir. Ainteratividadeaquise prende $\neq$ sprûprias limitaÁıes do ambiente que n,,o permite o uso de personagens eoroteiro fica, porenquanto, restrito ao percorrerdacidade virtual eoclube, bem como osjogos. Apesar das limitaÁıes doambiente, regularidadessensÛrio-motorasesimbÛlicas se produziam no espaÁo j- disponl̀vel. O desenvolvimentodosprojetosdeaprendizagem e sua publicaÁ,,o em forma de links na p.gina inicial da cooperativa, ampliar., semd 'vida, os espaÁos deinteratividade. Conhecendo ostrabalhos de outros estudantes haver-, ao menos potencialmente, um incremento da interatividade pelas trocas que ser,o possìveis.

Uma outra quest,,oque cabe ser ressaltada È a da familiariadade, $\mathrm{t}$, , marcante nesse ambiente. Paradoxalmente, deacordocomnossos resultados, o estranhamento foi necess.rio $\ddagger$ ludicidade e $\ddagger$ produÁ,, 0 de contextos de aÁ,,o. Essedadonosfazpensare relativizaros prÛprios depoimentos dos alunos, nos quais enfatizaram uma maior familiaridade. Cabe pensar que ambientes que estimulem a imers,,o e tambÈm a aprendizagem necessitamserambientesque perturbem, que estimulem a vontade deconhecer, explorar, interagir, conviver. AlĖmdisso, sensaÁ,,oderealidaden,,O advÈm necessariamente de uma reproduÁ,,O fiel dos objetos do mundo concreto, como crí- em os que advogam a simulaÁ,,o real como respons-velpelasensaÁ,,o de imers,,o. AcrenÁa na veracidade das interaÁıes no ambiente surge pelo efetivo acoplamento. A percepÁ,,oe os gestos vividos em uma realidade virtual podemdarnascimentoaregularidadessensÛriomotoras e simbÛlicas diferenciadas das que experimentamos na realidade concreta. A imers,,o È resultante, ent,,o, da construÁ,,o de novas regularidadesou acoplamentos.

Antes de tudo, pode ser interessante observar que os resultados se referem ao primeiro encontro dos sujeitos com os ambientes, enfim de seus modos iniciais de aÁ,,O. N,,O podemos dizer que a Cooperativa n, o È um ambienteimersivo. Contudo, pudemosapontar uma ṡ̀rie dequestıes que precisavam ser revistas , na construÁ,,o do ambiente, pois estas estavaminfluenciando no modo deoperardos sujeitoseestavamsendorestritivas $\ddagger$ interaÁ,,o, objetivo primordial doambiente.

Esses resultados permitem sustentar alteraÁıes e correÁıes nos rumos do desenvolvimento da Cooperativa. Um dado significativo desses resultados È o de propor a cooperativa para alĖm de um ambiente de encontro, como havlamos definido de inl̀cio, mas um ambiente de produÁ,,o, onde alunos e professores possam exercer sua autoria, influenciando nos rumos da prÛpria atualizaÁ,,o dacooperativa.

A sensaÁ,o de presenÁa nestes (imers,,o) ambientes, aliada $\ddagger$ sensaÁ,,odepertenÁaparticipativapelaautoria, ser-capazdeproduzirespaÁosdeaprendizagemquealÈmdeinovadores possam ser inusitados tanto para professores quanto para os estudantes. Estudos sobre as relaÁıes entre imersividade, autoria e produÁ,,odeaprendizagemprecisamserefetivados, uma vez que, como j- referimos, a imers,,o podeserumapossibilitadoradaaprendizagem, mas porsisÛn,,oagarante. 
Referíncias

ASSIS, Jesus de Paula. Roteiro em ambientes virtuais interativos. Cadernos da Pós Graduação. Campinas: UNICAMP. 1999. v. 3, n.1, p. 93-110.

CASAS, Luis Alberto Alfaro. Contribuições para a modelagem de um ambiente inteligente de educação baseado em realidade virtual. 1999. Disponl̀vel em: <http://www.eps.ufsc.br/teses99/casas/cap4a.html>. Acesso: 01 jul. 2003.

CHIC SOFTWARE. Rennes: Instituto de Matem tica da Universidade de Rennes, 1999, CD ROM Windows 1.1.

MACHADO, Arlindo. Máquina e Imaginário.S,,o Paulo: Edusp, 1996.

MARASCHIN, Cleci; MESQUITA, Jaqueline. Ambiente de Realidade Virtual Cooperativo de Aprendizagem: percepÁ,o em ambiente de realidade virtual. In: WRV - 3 rd Brazilian Workshop on Virtual Reality, 2000, Gramado. Anais. Gramado: Brazilian Computer Society, 2000, CD-ROM, p. 1-7.

MARASCHIN, Cleci et al. Avaliando a PercepÁ,,o em Ambientes de Realidade Virtual. In: XIII Sal,o de IniciaÁ,,o Cientìfica, 2001, Porto Alegre. Livro de Resumos. Porto Alegre: Universidade Federal do Rio Grande do Su/PROPESQ, Porto Alegre, 2001 , p. 579

MARASCHIN, Cleci; ZANIOL, Elis,ngela. Avaliando a PercepÁ„,o em Ambientes de Realidade Virtual ñ Projeto ARCA. In: 53a Reuni,,o Anual da SBPC, 2001, Salvador. Anais. Salvador: Sociedade Brasileira para o Progresso da Cíncia. 2001. p.124

MARASCHIN, Cleci; ZANIOL, Elis,ngela. Imers,„o e Autoria em Ambientes Virtuais Cooperativos. XIV Sal,,o de IniciaÁ„,O Cientìfica, 2002, Porto Alegre. Anais. Porto Alegre: Sal,o de IniciaÁ„, Cientìfica - Universidade Federal do Rio Grande do Sul.2002. p. 773.

MATURANA, Humberto. El sentido de lo humano. Santiago: Dolmen, 1994.

Recebido em 01/09/2003

AceitoparapublicaÁ,,oem07/07/2004

\section{Cleci Maraschin}

Doutora, Professora do Programa de PÛs GraduaÁ,,O em Psicologia Social e Institucional - UFRGS e do Programa de PÛs GraduaÁ,,o em Psicologia Social e Institucional - UFRGS. Professora do ProgramadePÛs GraduaÁ,,ode Inform·ticanaEducaÁ,,o.

E-mail:clecim@psico.ufrgs.br

\section{Elis,ngela Zaniol}

PsicÛloga, Mestranda em Psicologia Social e InstitucionalUFRGS.

E-mail:elisangelaz@yahoo.com.br 